\title{
Time series analysis with the VSAA method
}

\author{
S. Tsantilas, ${ }^{1}$ K. Kolenberg, ${ }^{2}$ and H. Rovithis-Livaniou ${ }^{1}$ \\ ${ }^{1}$ Dept. of Astrophysics, Astronomy \& Mechanics, Faculty of Physics, Athens University, \\ Panepistimiopolis, Zografos 157 84, Athens, Greece \\ 2 Institut für Astronomie, Universität Wien, Türkenschanzstrasse 17, 1180 Vienna, Austria
}

\begin{abstract}
Time series analysis is a common task in many scientific fields, and so it is in astronomy, too. Fourier Transform and Wavelet Analysis are usually applied to handle the majority of the cases. Even so, problems arise when the time series signal presents modulation in the frequency under inspection. The Variable Sine Algorithmic Analysis (VSAA) is a new method focused exactly on this type of signals. It is based on a single sine function with variable coefficients and it is powered by the simplex algorithm. In cases of phenomena triggered by a single mechanism - that Fourier Transform and Wavelet Analysis fail to describe practically and efficiently - VSAA provides a straightforward solution. The method has already been applied to orbital period changes and magnetic field variations of binary stars, as well as to the Blazhko effect of the pulsating RR Lyrae stars and to sunspot activity.
\end{abstract}

Individual Objects: CG Cyg, RR Lyr

\section{Introduction}

Research on astronomical data often involves time series analysis. This also holds for time series of pulsating stars showing modulation, of orbital period variations in binary systems, of sunspot or starspot activity, etc. The analysis of these time series is usually carried out using the well-known Fourier transform. The problem that arises in the majority of these cases is that the modulation of the phenomena is not strictly periodic but more of a quasiperiodic nature. Because the Fourier transform is focused on the frequency domain only, the micro-variations in amplitude and frequency through time are untraceable with this method.

\section{The VSAA method}

The VSAA - Variable Sine Algorithmic Analysis - (Tsantilas \& Rovithis 2007) is an method incorporated in a computer code and powered by the Simplex algorithm (Nelder \& Mead 1965). It is based on a single sine function with coefficients that are not constant but are functions of time. Instead of having a large number of frequencies needed to explain the signal (as in Fourier and Wavelet analysis), the VSAA traces the micro-variations of a single frequency more or less continuously. Therefore, the output of the analysis is simple and clear, while the results can be assigned in a straightforward manner to a single mechanism that modulates its characteristics (amplitude and frequency) through time. The core function is:

$$
f(t)=a \cdot \sin (b \cdot t+c),
$$

where $a=a(t), b=b(t)$ and $c=c(t)$, i.e. they are functions of time. 


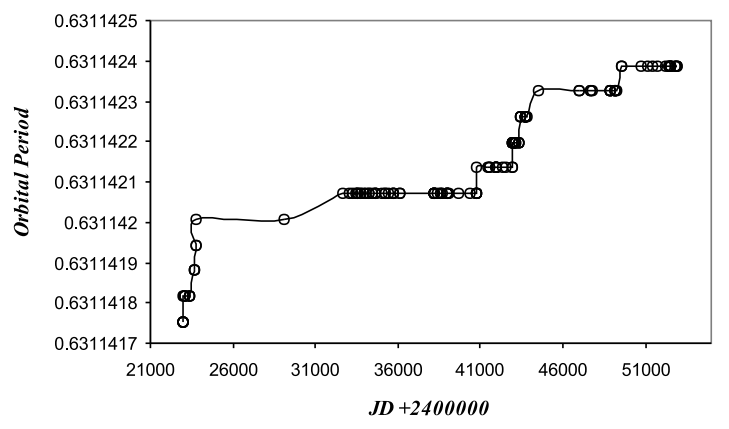

Figure 1: Orbital period variation of CG Cyg.

The user has to define some initial values for the amplitude, the starting frequency and the (possible) phase shift of the signal, the sliding window width, the number of iterations for every step of the simplex, and the accuracy threshold of the simplex.

The three major parameters: frequency, amplitude and phase shift, can be defined independently as adjustable or fixed. Because the frequency and phase shift are connected, it is suggested to fix one of them. After the initiation of the starting values the program performs a windowed partial fit using the simplex algorithm. This procedure continues automatically until the end of the time series data set under inspection. The output of the analysis is a set of $N-\ell$ vectors $v_{j}$, where $N$ is the number of the input data points and $\ell$ is an internal parameter which secures that there are enough points in order to get a decent fit. The vectors have the form: $v_{j}=\left(t_{j}, a_{j}, b_{j}, c_{j}, \sigma_{j}, F_{j}\right), j=1 \ldots N-\ell$, where $t_{j}$ denotes the time, $a_{j}(t)$ is the amplitude of the signal, $b_{j}(t) / 2 \pi=f(t)$ denotes the variable frequency, $c_{j}(t)$ stands for the phase shift of function $(1), \sigma_{j}=\sqrt{\frac{\sum\left(s_{j}-F_{j}\right)^{2}}{w}}$ is the mean error, where $s_{j}$ is the original signal data and $F_{j}$ is the VSAA fit to the signal.

\section{Applications}

The VSAA has already been applied to synthetic data (Tsantilas \& Rovithis 2008b), to the analysis of the Blazhko effect (Kolenberg \& Tsantilas 2008) and to sunspot data (Reindel et al. 2008, Tsantilas \& Rovithis 2008a). By fixing the amplitude to an arbitrary value, the VSAA could also trace the micro-variations of the actual period of a system in the time-frequency domain. Here we present such a first application: the extraction of the orbital period of the eclipsing binary CG Cyg directly from its times of minima (Fig. 1). It is important to notice here that the period can be acquired without the involvement of the $\mathrm{O}-\mathrm{C}$ (observed minus calculated time of minima) diagram.

\section{References}

Kolenberg, K., \& Tsantilas, S. 2008, CoAst, 157, 52

Nelder, J. A., \& Mead, R. 1965, Comput. J., 7, 308

Reindel, A., Bradley, P., Tsantilas, S., \& Guzik, J. 2008, GONG 2008/SOHO XXI conference, Boulder, Colorado

Tsantilas, S., \& Rovithis-Livaniou, H. 2007, arXiv:0709.3224

Tsantilas, S., \& Rovithis-Livaniou, H. 2008a, Rom. Astron. Journal, in press

Tsantilas, S., \& Rovithis-Livaniou, H. 2008b, CoAst, 157, 87 